\title{
Protée
}

\section{Nous - infinis entre deux fins}

\section{Michel Lisse}

Volume 27, numéro 3, 1999

L'imaginaire de la fin

URI : https://id.erudit.org/iderudit/030575ar

DOI : https://doi.org/10.7202/030575ar

Aller au sommaire du numéro

Éditeur(s)

Département des arts et lettres - Université du Québec à Chicoutimi

\section{ISSN}

0300-3523 (imprimé)

1708-2307 (numérique)

Découvrir la revue

\section{Citer cet article}

Lisse, M. (1999). Nous - infinis entre deux fins. Protée, 27(3), 103-108. https://doi.org/10.7202/030575ar

\section{Résumé de l'article}

Dans cet article, je tente d'étudier l'oscillation entre les concepts de fini et d'infini dans divers textes de Jacques Derrida et son incidence sur les problématiques de la promesse et de la mort. Cette étude me conduit à considérer une proposition de Jacques Derrida sur un " nous infini » face au " soleil fini » comme une réécriture du Soleil placé en abîme de Francis Ponge. d'utilisation que vous pouvez consulter en ligne.

https://apropos.erudit.org/fr/usagers/politique-dutilisation/ 


\section{NOUS - IN FIN IS ENTRE DEU X FIN S}

MICHEL LISSE*

Nous sommes peut-être entre ces deux veilles qui sont aussi deux fins de l'homme. Mais qui, nous?

Jacques Derrida

Dans un texte peu connu de Jacques Derrida, quelques phrases paraissent bien énigmatiques même si leur auteur a jugé utile de les faire précéder d'un commentaire qui aide à la compréhension. Ce passage extrait de «Demeure, Athènes» résiste à l'interprétation:

Nous sommes infinis, donc soyons infinis, éternellement. [...] Le soleil même est fini, nous le savons,

et sa lumière un jour peut prendre fin, mais nous? Laissons la finitude au soleil [...] il n'y a de deuil, et de mort [...] que pour ce qui regarde le soleil. ${ }^{1}$

Mon propos, dans cet article, sera de tenter d'entamer une lecture de ces phrases dans la perspective qui est celle de ce numéro de Protée.

Dans son «Derridabase», Geoffrey Bennington - ou, plus exactement, un interlocuteur dans un dialogue (celui qui paraît être en position magistrale) concède le point suivant:

Il est certain que les termes "fini» et «infini» fonctionnent de façon déroutante dans les textes de

Derrida. $^{2}$

Christopher Johnson, quant à lui, a consacré un sous-chapitre de son System and Writing in the Philosophy of Jacques Derrida ${ }^{3}$ à cette problématique de l'infini. Il a jugé utile de rappeler que Derrida, à l'occasion de son premier commentaire de Levinas («Violence et métaphysique»), a qualifié d'étrange l'idée de l'Infini ${ }^{4}$. Mieux, Christopher Johnson a placé en exergue une phrase tirée du même commentaire de Levinas où Derrida insiste sur l'impossibilité pour un système d'être fini ou infini: 
Un système n'est ni fini ni infini. Une totalité structurale échappe en son jeu à cette alternative. Elle échappe à l'archéologie et à l'eschatologie et les inscrit en elle-même. ${ }^{5}$

À quoi tient cette difficulté mise en évidence par Geoffrey Bennington et Christopher Johnson? Tout d'abord en ceci que l'opposition du fini et de l'infini est une opposition métaphysique, qui, comme toutes les oppositions métaphysiques, se déconstruit, comporte en son sein une aporie. Ensuite parce que Derrida, bien évidemment conscient de cette précarité de la différence entre le fini et l'infini, n'a cesse de recourir malgré tout à ces deux mots, par le biais, pour reprendre le terme de Bennington, d'un usage déroutant. Enfin, il faut l'avouer, peut-être sommesnous plus sensibles, plus interpellés par des questions touchant à la fin - donc à notre ou nos fin(s) - que par d'autres questions? Dans «Les fins de l'homme», Derrida écrit que «l'homme est ce qui a rapport à sa fin, au sens fondamentalement équivoque de ce mot", c'est-à-dire à la fois le telos, la finalité et la mort:

Depuis toujours, la fin transcendantale ne peut apparaître et se déployer qu'à la condition de la mortalité, d'un rapport à la finitude comme originalité de l'idéalité. Le nom de l'homme s'est toujours inscrit dans la métaphysique entre ces deux fins. Il n'a de sens que dans cette situation eschato-téléologique. ${ }^{6}$

Pour tenter de poursuivre la réflexion sur cette triple difficulté, je vais me risquer à aborder quelques textes de Jacques Derrida. Délibérément, je ferai l'impasse sur des textes, ô combien importants, mais déjà commentés par Bennington et Johnson, comme L'Écriture et la Différence, De la Grammatologie, La Voix et le phénomène, pour me tourner vers quelques textes plus récents qui relancent et déploient les questions relatives au fini, à l'infini et à la mort.

Dans "Avances", Jacques Derrida met au jour ce qu'il nomme «une aporie intrinsèque du concept de promesse": une promesse doit être, «doit toujours être à la fois, en même temps, infinie et finie dans son principe» 7 . Pourquoi? Une promesse ne peut être certaine, sûre, une promesse n'a de sens et d'effet que si l'on promet ce dont on n'a aucune certitude. Si je promets à une femme de l'aimer toute ma vie, cette promesse ne vaut que parce qu'il y a le risque que je ne la tienne pas et parce que je ne sais pas si je tiendrai ma promesse. Je n'ai et je ne peux avoir aucune certitude à ce sujet. Au moment où je promets, j'espère tenir ma promesse, mais cette espérance ne peut se régler sur aucun calcul. C'est pourquoi une promesse est «infinie parce qu'elle doit pouvoir se porter au-delà de tout programme possible, et qu'à ne promettre le calculable et le certain on ne promet plus " 8 . Mais ma promesse doit également être finie, car une promesse, sans cesse rompue et toujours répétée, n'en est plus une: «à promettre l'infini à l'infini on ne promet plus rien de présentable, donc on ne promet plus». L'aporie du concept de promesse s'énonce de la sorte: «pour être une promesse, une promesse doit pouvoir être intenable et donc pouvoir ne pas être une promesse (car une promesse intenable n'est pas une promesse)» 9 . L'usage de l'italique signifie qu'il s'agit dans le cas de la promesse d'une potentialité nécessaire, mais non nécessairement actualisée.

Cette réflexion sur l'aporie du concept de promesse conduit Derrida à s'interroger sur un énoncé à la "grammaire singulière». Que signifie l'énoncé "nous nous promettons»? Derrida pose une série de questions:

Nous nous promettons quoi? Nous? À qui? À quoi? À nous? À

l'autre? Serions-nous promis (comme une chose promise, en quelque sorte: chose promise, chose due), à la fois gardiens et garants, héritiers ou sujets d'une promesse intenable? D'une dette insolvable? 10

Dans une telle promesse, quand je me promets à moi-même, quand je me promets moi-même à moimême, la première personne, dit Derrida, est déjà plurielle. Elle est divisée par l'altérité; à vrai dire constituée par cette altérité sans laquelle aucune promesse ne serait possible:

La pensée de la promesse ou du promettre suppose [...] «une première personne». [...] Mais elle suppose aussi que cette première personne soit du pluriel, plus d'une, l'une et l'autre. Même quand je me promets, que je me promette ceci ou cela ou 
que je me promette moi-même à moi-même, l'autre est déjà dans la place. Il faut lui faire place car il n'y a pas de place sans lui. Ce qui est indérivable, à cette place, c'est le «nous» de l'un et de l'autre, de l'un comme l'autre, même quand l'un se garde de l'autre. 11

Ce qui vaut pour «je me promets» vaut également pour «nous nous promettons». L'autre, l'autre «nous», est inanticipable, inconnaissable, irréductible à un quelconque savoir:

Nous ne savons pas ce que nous (nous) promettons quand ce que nous nous promettons à nous-mêmes, c'est «nous». 12

Cette béance originaire du «je» (qu'il ne faut pas identifier au sujet), infiniment ouvert à l'autre, ouvert par l'autre, et par là infini, Jacques Derrida la retrouve chez Emmanuel Levinas. Dans Adieu, étudiant les motifs de l'accueil, de l'hospitalité dans les textes de celui-ci, Derrida n'a cesse de rappeler que le rapport à l'autre suppose une interruption infinie, une séparation infinie, que l'intentionnalité est d'abord ouverture à l'infini de l'autre, à l'infini comme autre, toujours déjà pré-originairement accueilli. Si nous possédons l'idée d'infini, c'est parce que nous avons déjà accueilli Autrui ${ }^{13}$, dès lors toute éthique ou philosophie première, pour reprendre des mots de Levinas, est expérience de l'hospitalité "parce qu'elle s'ouvre, pour l'accueillir, à l'irruption de l'idée d'infini dans le fini», "hospitalité d'un seuil fini qui s'ouvre à l'infini»" ${ }^{4}$. De ce point découle une «définition» de la conscience comme hospitalité, accueil infini. La problématique de l'infini dans la finitude se retrouve autrement dans l'œuvre de Martin Heidegger.

Dans Apories, Jacques Derrida examine en effet le rapport établi par celui-ci entre le Dasein et la mort. Pour permettre la compréhension de ce qui va suivre, je rappelle, après Derrida, que Heidegger distingue le mourir (Sterben), propre au Dasein, du périr (Verenden) comme fin du vivant en général (y compris de l'homme que Heidegger doit radicalement distinguer du Dasein), avec une modalité intermédiaire qui suppose le Sterben, à savoir le décès (Ableden), accompagné de ses connotations médico-légales, lui aussi propre au Dasein. C'est pourquoi Heidegger peut soutenir la proposition suivante:

Le Dasein ne périt jamais. Mais le Dasein ne peut toutefois décéder que pour autant qu'il meurt. 15

Si le Dasein ne périt jamais, cela signifie, d'une certaine façon, qu'il n'est pas confronté à cette modalité du finir, à ce Verenden. En tant que Dasein, le Dasein ne finit pas:

Le Dasein, le Dasein en tant que tel, ne connaît pas de fin au sens de verenden. De ce point de vue du moins et en tant que

Dasein, je suis sinon immortel du moins impérissable: je ne finis pas, je n'en finis jamais, je sais que je ne prendrai pas fin. 16

Si Jacques Derrida va, dans la suite de sa conférence, pointer toutes les apories de la pensée de la mort chez Heidegger, s'il va montrer que les distinctions entre le périr, le mourir et le décéder ne vont pas sans se contaminer, il gardera l'idée d'un «nous» traversé d'infini. La dernière phrase d'Apories est, sur ce point précis, explicite: «... nous serions sans cesse plus jeunes et plus vieux, en un dernier mot infiniment finis".

Avant de revenir à la citation initiale, il importe de rappeler très brièvement quelques points mis en lumière par D'un ton apocalyptique adopté naguère en philosophie. Ce que montre Jacques Derrida dans cette conférence, c'est que, d'une certaine manière, tout texte devient apocalyptique dès que les voix se démultiplient, dès que l'on n'est plus assuré de savoir qui parle ou écrit. D'où la question de savoir si l'apocalyptique ne serait pas «une condition transcendantale de tout discours, de toute expérience même, de toute marque ou de toute trace " 17 . Autrement dit, n'y aurait-il pas une «structure apocalyptique» du langage ou de l'écriture, prise entre deux fins? Car la dénonciation du discours apocalyptique qui doit être menée aussi loin que possible - et Derrida insiste à plusieurs reprises sur l'importance de ce geste, notamment du point de vue politique - est elle aussi apocalyptique. Autre aporie 
qui oblige à mettre en œuvre une logique scripturale du X sans X, une "apocalypse sans apocalypse», c'est-àdire "sans vision, sans vérité, sans révélation " 18 , qui dit une fin sans fin.

La citation initiale de ce texte peut maintenant être abordée. «Demeure, Athènes» est parcouru par une phrase, à vrai dire la première phrase qui ne cesse de revenir hanter le texte. Cette phrase semble dire un certain devoir ou une certaine dette que nous aurions à l'égard de la mort. "Nous nous devons à la mort», voilà la phrase qui, incessamment, fait retour de manière littérale ou autre. Il faut attendre la fin du texte pour que cette sentence soit expliquée par Derrida qui va alléguer une intraductibilité de celle-ci et dès lors récuser deux traductions possibles.

1. La traduction qui recourrait à la "grande tradition post-socratique et sacrificielle de l'être-pour-la-mort» ${ }^{19}$ et proposerait d'entendre la phrase dans une perspective éthique: il importe de se dévouer à la mort à laquelle nous sommes destinés, il faut s'en soucier, y ordonner nos projets, notre vie, économiser celle-ci...

2. La traduction, déjà moins traditionnelle, qui prendrait en compte la dette, le devoir, l'obligation... Nietzsche, Heidegger et Levinas pourraient nous aider à entendre "nous nous devons à la mort» dans cette perspective. Jacques Derrida montre qu'il est possible de traduire la phrase "nous nous devons à la mort» en accentuant, c'est là le paradoxe, son intraductibilité grammaticale: comment rendre dans une autre langue le redoublement du "nous»? Le second «nous» ferait de "nous», du premier «nous», dans une allusion à Levinas, un "gage» ou un «otage»:

[...] le premier nous, le «sujet", viendrait après le second (objet réfléchissant [...]). Il ne se constituerait comme «sujet» qu'après avoir réfléchi le «second» «nous» constitué, lui, en «objet» dû: «nous» sommes «dus» $[. .$.$] , nous nous rapportons à nous-mêmes,$ nous nous prenons en vue comme un dû, pris dans une dette ou un devoir qui nous précède et nous institue, une dette qui nous contracte avant même que nous ne l'ayons contractée. 20

Au nom de quoi Derrida peut-il récuser ces deux traductions qui, pourtant, il vient de le préciser, sont possibles? L'intraductibilité grammaticale ne paraît pas être un argument suffisamment puissant comme le montre Derrida lui-même puisqu'il donne à ses lecteurs deux pistes de traduction. Par contre ce que Derrida nomme «la modalité pragmatique de son événement", à savoir le surgissement de la phrase seule, décontextualisée, le surprenant dans le soleil (c'est ce qu'il raconte au début du livre), peut permettre une autre compréhension de "nous nous devons à la mort». Il pourrait s'agir d'une «protestation indignée» contre les deux traditions qui avancent que "nous nous devons à la mort", d'un refus de la dette prétendument originaire, de la «religion du deuil», de la "culture de la perte et du manque, etc.». Protestation d'un «nous» contre l'autre:

Nous nous devons à la mort, il y a bien un nous, le second qui se doit ainsi, mais nous, en premier lieu, non, le premier nous qui regarde, observe et photographie l'autre, et qui parle ici, c'est un vivant innocent qui à jamais ignore la mort en ce nous nous sommes infinis [...]. Nous sommes infinis, donc soyons infinis, éternellement. [...] Le soleil même est fini, nous le savons, et sa lumière un jour peut prendre fin, mais nous? Laissons la finitude au soleil [...] il n'y a de deuil, et de mort [...] que pour ce qui regarde le soleil. 21

Entendre la formule «nous nous devons à la mort» sous le mode de la dénonciation serait, je risque l'hypothèse, une manière pour Derrida de réécrire Le Soleil placé en abîme de Francis Ponge. Pour déplier quelque peu cette hypothèse, il est nécessaire de se reporter à Signéponge 22 et plus précisément aux pages 112 à 115 . Derrida commence par préciser que le soleil, «l'antique philosophème», paraît être unique, parce qu'il est un référent "irremplaçable», «sans substitut possible». Cette précision se termine par une allusion à Platon qui a pensé le soleil comme «source et condition de tout objet visible ou nommable" et donc comme au-delà de l'être (epekeina tes ousias). À plusieurs reprises, Jacques Derrida s'est intéressé à ce passage de La République où Platon, devançant toute la tradition de la théologie négative, pense un au-delà de la présence, de l'étantité, voire de 
l'être ${ }^{23}$. Une parenthèse de «La mythologie blanche» 24 décrit le paradoxal statut que Platon accorde au soleil :

(Dans la République [VI-VII], [...], le soleil paraît. Pour disparaître. Il est là, mais comme la source invisible de la lumière, dans une sorte d'éclipse insistante, plus qu'essentielle, produisant l'essence - être et paraître - de ce qui est. On ne peut le regarder en face sous peine d'aveuglement et de mort. Se tenant au-delà de ce qui est, il figure le Bien dont le soleil sensible est le fils: source de vie et de visibilité, de semence et de lumière.)

Si le soleil a un côté spectral, s'il paraît pour disparaître, s'il s'éclipse sans cesse, il faut conclure qu'il «n'appartient plus, par position transcendantale (epekeina tes ousias), au système qu'il rend possible» ${ }^{25}$. Cette conclusion en génère une autre: il échapperait à toute mise en texte, à toute mise en abyme parce qu'il est un trou, l'abîme métaphysique. Francis Ponge, dans Le Soleil placé en abîme, résume la pensée platonicienne de la sorte:

Le PLUS BRILLANT des objets du monde n'est - de ce fait non - n'est pas un objet; c'est un trou, c'est l'abîme métaphysique: la condition formelle et indispensable de tout au monde. La condition de tous les autres objets. La condition même du regard. 26

Le soleil est un abîme impossible à mettre en abyme.

Et pourtant, Ponge parvient à détrôner ce tyran, à le coucher, à le mettre sur la page, à le transformer en une «putain rousse»... Pour rappel, quelques extraits commentés ou paraphrasés du Soleil placé en abîme, non sans rapport, je l'espère, avec mon propos. «Le soleil anime un monde qu'il a d'abord voué à la mort» et dans ce monde, le soleil qui a déjà perdu de son pouvoir, qui est «dans les derniers temps de son pouvoir", va créer «des êtres capables de le contempler", ces êtres (Ponge ne parle pas encore d'hommes, d'animaux, de végétaux, de minéraux...) «meurent tout à fait», cependant cette mort ne les empêche pas de continuer «leur ronde» au service du soleil comme «spectateurs (ou gens d'escorte)». Mais ces êtres sont capables de compter «les myriades d'autres soleils», de situer «leur propre soleil parmi l'infinité des astres, non comme le plus important", mais comme «l'un seulement des soleils». Intervient alors un «je» qui va proclamer sous le mode de la négation la vengeance de ces êtres: «Et je ne dis pas qu'une telle considération les rassure, mais elle les venge». Parmi ces êtres qui comptent le soleil, il faut compter l'homme qui va aller au-delà du calcul, plus exactement l'abandonner:

Ainsi, plongé dans le désordre absurde et de mauvais goût du monde, dans le chaos inouï des nuits, l'homme du moins compte les soleils.

Mais enfin, son dédain s'affirme et il cesse même de les compter. 27

Un «nous» a proclamé la fin du soleil et a affirmé son dédain pour une dette à l'égard de la mort. Un «nous» a dénoncé de manière emportée au moins deux traditions philosophiques, la tradition postsocratique et la tradition nietzschéo-heideggerrianolevinassienne. Mais cette dénonciation, se demande Jacques Derrida, «n'est-ce pas le dernier signe de deuil, la stèle la plus ensoleillée, la dénégation la plus conséquente $[\ldots]$ »28?

Le dernier mot à propos de la fin - une dénégation?

\section{N O TES}

1. J. Derrida, «Demeure, Athènes (Nous nous devons à la mort)", dans Athènes à l'ombre de l'Acropole, photographies de J.-F. Bonhomme, Athènes, Olkos, 1996, p. 63.

2. G. Bennington, «Derridabase», dans G. Bennington et J. Derrida, Jacques Derrida, Paris, Le Seuil, 1991, p. 111.

3. Cambridge, Cambridge University Press, 1993.

4. Cf. J. Derrida, L'Écriture et la Différence, Paris, Le Seuil, 1967, p. 146.

5. Ibid., p. 180.

6. J. Derrida, Marges. De la philosophie, Paris, Minuit, 1972, p. 147.

7. J. Derrida, «Avances», dans S. Margel, Le Tombeau du dieu artisan, Paris, Minuit, 1995, p. 26.

8. Ibid.

9. Ibid. 
10. Ibid., p. 36 .

11. Ibid., p. 40

12. Ibid., p. 39.

13. Cf. J. Derrida, Adieu. À Emmanuel Levinas, Paris, Galilée, 1997, p. 21, 51, 60 .

14. Ibid., p. 88 .

15. M. Heidegger cité par J. Derrida, Apories. Mourir - s'attendre aux

"limites de la vérité», Paris, Galilée, 1996, p. 73.

16. Ibid., p. 76.

17. J. Derrida, D'un ton apocalyptique adopté naguère en philosophie, Paris, Galilée, 1983, p. 77-78.

18. Ibid., p. 95.

19. J. Derrida, "Demeure, Athènes (Nous nous devons à la mort)», op. cit., p. 61 .

20. Ibid., p. 61-62.

21. Ibid., p. 63 .
22. Paris, Le Seuil, 1988.

23. Cf. par exemple J. Derrida, La Dissémination, Paris, Le Seuil, 1972, p. 193.

24. Cf. Marges, op. cit., p. 289.

25. Signéponge, op. cit., p. 113. Il faudrait étudier toutes les pages consacrées par Jacques Derrida au soleil dans la tradition

philosophique. Aux noms de Platon, d'Aristote, de Kant et de Hegel, vient s'ajouter celui de Nietzsche qui pense le midi comme moment sans ombre, moment d'affirmation, de «neutralité démonique [...], délivrée du négatif et du dialectique "(Otobiographies, Paris, Galilée, 1984, p. 65).

26. Cité par J. Derrida, Signéponge, op. cit., p. 113.

27. F. Ponge, Euvres complètes I, sous la dir. de B. Beugnot, Paris, Gallimard, 1999, p. 783.

28. J. Derrida, "Demeure, Athènes (Nous nous devons à la mort)", op. cit., p. 63 . 